\begin{tabular}{llll} 
Abstract 127 Table 1 & (Investigations) & & \\
\hline Investigation & Case 1 & Case 2 & Case 3 \\
\hline Haemoglobin (g/L) & 93 & 96 & 75 \\
White cell counts (IL) & $9.5 \times 10^{6}$ & $3.5 \times 10^{6}$ & $4.8 \times 10^{6}$ \\
Lymphocyte count (/L) & $1.9 \times 10^{6}$ & $1.2 \times 10^{6}$ & $0.3 \times 10^{6}$ \\
Platelet count (/L) & $145 \times 10^{9}$ & $147 \times 10^{9}$ & $37 \times 10^{9}$ \\
Total serum bilirubin (mg/dl) & 13.8 & & 7.1 \\
Conjugated bilirubin (mg/dl) & 12 & & 4 \\
Aspartate aminotransferase (IU/L) & 329 & 203 & 69 \\
Alanine aminotransferase (IU/L) & 96 & 156 & 57 \\
Urine protein (mg/m²/hour) & & $24 \mathrm{mg} / \mathrm{m}^{2} / \mathrm{hr}$ & \\
C3 (Normal 50-150 mg/dL) & 68 & 23 & 68 \\
C4 (Normal 20-50 mg/dL) & 4 & 4 & 3 \\
ANA & $3+$ diffuse & $3+$ diffuse & $4+$ speckled \\
Anti dsDNA (N:<60 IU/mL) & 733 & 1621 & 689 \\
\hline
\end{tabular}

presenting manifestation is uncommon in children with lupus.

Methods We retrospectively studied 140 pediatric lupus patients from January 1993- November 2018 and collected clinical and laboratory data of patients (3) who had liver involvement as presenting manifestation.

Results Case 1 was 13-year-old girl with fever and joint pain for 7 months associated with rash and yellow discoloration of eyes and body for 1 month. She also had altered behavior for 3 days. Examination revealed malar rash, pallor, jaundice, bilateral knee arthritis and hepatosplenomegaly. Investigations revealed (table 1) anemia, thrombocytopenia and lymphopenia. She had conjugated hyperbilirubinemia. Transaminases were elevated. Further investigations confirmed diagnosis of lupus (table 1). Markers for autoimmune hepatitis (SMA; LKM) were negative. She received pulse methylprednisolone followed by tapering doses of oral prednisolone. In view of neurological involvement she also received cyclophosphamide and shifted to azathioprine later. Her initial SLEDAI-2k was 22, that decreased to 4 at 2 year follow-up.

Case 2 was a 11-year-old boy with fever, rash and joint pain for 1 month. Examination revealed pallor, malar rash, oral ulcers, arthritis of bilateral elbow and knee and hepatomegaly. Investigations (table 1) revealed anemia; thrombocytopenia; leucopenia and lymphopenia. In view of multisystem involvement a possibility of lupus was considered which was confirmed by investigations (table 1). He had elevated transaminases. There was no coagulopathy. He was started on oral prednisolone. She also had class 3 lupus nephritis and received pulse cyclophosphamide followed by azathioprine in maintenance. Her transaminases decreased in follow-up. Her initial SLEDAI-2k was 18 , that decreased to 2 at 2 year follow-up.

Case 3 was an 9-year-old girl who had rash and abdominal distention for 20 days and fever and altered sensorium for 6 days. On examination she had pallor, malar rash, ascitis and hepatosplenomegaly. Investigations revealed anemia; thrombocytopenia; leucopenia and lymphopenia. She had conjugated hyperbilirubinemia, elevated transaminases and coagulopathy. She also had deranged renal function tests. Ultrasonography reveals coarse echotexture, gross ascitis and splenomegaly. Possibility of lupus with acute on chronic liver decompensation was considered which was confirmed by investigations (table 1). She was given methyl prednisolone. She had progressive azotemia so possibility of rapidly progressive renal failure was considered and cyclophosphamide was given. However, she developed refractory shock and eventually expired.

Conclusions Hepatic involvement at presentation in lupus can be multifaceted and poses challenge in diagnosis.

Funding Source(s): None

\section{THE LUPUS SEVERITY INDEX IS A PREDICTOR OF DAMAGE AND DEATH IN LUPUS PATIENTS}

${ }^{1}$ Paul R Fortin*, ${ }^{2}$ Anne-Sophie Julien, ${ }^{3}$ Ann E Clarke, ${ }^{4}$ Janet Pope, ${ }^{5}$ Mark Matsos, ${ }^{6}$ Douglas Smith, ${ }^{7}$ Christine Peschken. ${ }^{1} \mathrm{CHU}$ de Québec - Université Laval; ${ }^{2}$ Université Laval; ${ }^{3}$ Division of Rheumatology, Cumming School of Medicine, University of Calgary: ${ }^{4}$ University of Western Ontario; ${ }^{5}$ McMaster University; ${ }^{6}$ University of Ottawa; ${ }^{7}$ Faculty of Medicine, Department of Internal Medicine, University of Manitoba

\subsection{6/lupus-2019-Ism. 128}

Background Predictors of poor outcome in systemic lupus erythematosus (SLE) may lead to the identification of high-risk patients at the onset of disease (incident cases) and/or when we first assess them in our clinics (prevalent cases). We tested whether the Lupus Severity Index (LSI) can help characterize high versus low risk lupus patients.

Methods Population: Patients from six lupus centers were recruited according to a standard data collection protocol. We characterized incident cases and prevalent cases as those with a diagnosis made within or after the previous 15 months. Data collected: Demographic, socioeconomic, disease specific and medication data were collected at baseline and annually. We collected: the American College of Rheumatology (ACR) and the Systemic Lupus International Collaborating Clinics (SLICC) classification criteria, the SLE Disease Activity Index (SLEDAI), the Systemic Lupus Activity Questionnaire (SLAQ), and the SLICC Damage Index (SDI). The LSI was derived from the ACR classification criteria and used as a predictor variable. Statistical analyses: Kruskal-Wallis test and Spearman correlations were used to see the association of LSI with categorical and continuous variables respectively. The baseline LSI was used to predict outcomes at follow-ups using logistic regressions and Spearman correlations for dichotomous and continuous variables respectively.

Results We enrolled 639 lupus patients and 440, 324 and 168 were re-evaluated at 1,2 and 3 years. Baseline characteristics (table 1) [median (IQR)] were: age $=49.0 \quad(36.8-$ 58.5 ) years, female $=92 \%$, Caucasian $=74 \%$, disease duration=10.1 (2.7-20.6) years. We had 129 (20\%) incident cases and 471 (74\%) prevalent cases with missing information in 39 (6\%). Twelve patients died during follow-up. Table 1 summarizes baseline associations between LSI and several characteristics for the incident and prevalent cases. We found that age, sex, ethnicity (Asian worse LSI), SLICC classification criteria, SLEDAI, prednisone use and daily dose were associated with LSI in both incident and prevalent groups while the SDI and the use of immunosuppressors drugs was associated with LSI only in the prevalent cases. In follow-up, baseline LSI predicted SDI in prevalent cases $(p=0.02)$ with a trend in incident cases $(p=0.07)$. LSI predicted death in the prevalent group.

Conclusions The LSI is easy to derive from the ACR classification criteria and a useful measure of severity in lupus. The LSI is associated with baseline characteristics, some of them - 
Abstract 128 Table 1 Patients characteristics and associations with LSI by disease duration, at baseline

\begin{tabular}{|c|c|c|c|}
\hline \multirow{2}{*}{$\begin{array}{l}\text { Variables * } \\
\text { Patient's characteristics }\end{array}$} & \multirow{2}{*}{$\begin{array}{l}\text { Descriptive } \\
\text { Statistics ** }\end{array}$} & \multicolumn{2}{|c|}{ Association with LSI *** } \\
\hline & & Incident cases & Prevalent cases \\
\hline Age & $\begin{array}{l}49.0 \\
(36.8-58.5)\end{array}$ & $-0.19(p=0.028)$ & $-0.19(p=<0.001)$ \\
\hline Gender & & $p=0.007$ & $p=0.006$ \\
\hline Women & $589(92)$ & $5.4(4.9-6.3)$ & $6.3(5.7-8.0)$ \\
\hline Men & $55(9)$ & $6.7(5.6-8.3)$ & $7.1(5.8-8.5)$ \\
\hline Ethnicity ( $n=592)$ & & $p=0.032$ & $p=0.005$ \\
\hline Caucasian & $437(74)$ & $5.4(4.7-6.4)$ & $5.7(5.1-7.9)$ \\
\hline Asian & $80(14)$ & $6.1(5.4-8.5)$ & $7.7(5.3-8.2)$ \\
\hline Other & $75(13)$ & $5.9(5.3-8.1)$ & $5.9(5.2-8.2)$ \\
\hline Marital Status $(n=586)$ & & $p=0.318$ & $p=0.076$ \\
\hline Single & $107(18)$ & $5.7(5.3-7.3)$ & $6.5(5.3-8.2)$ \\
\hline Married & $387(66)$ & $5.4(4.7-6.3)$ & $5.8(5.2-8.0)$ \\
\hline Other & $92(16)$ & $5.4(4.8-7.1)$ & $5.7(4.9-8.2)$ \\
\hline Site & & $p=0.789$ & $p=0.383$ \\
\hline Winnipeg & 231 (36) & $5.8(4.9-7.0)$ & $5.6(5.0-7.8)$ \\
\hline Hamilton & $25(4)$ & $6.2(5.4-6.6)$ & $6.6(5.4-7.1)$ \\
\hline Quebec & $129(20)$ & $5.4(4.6-6.8)$ & $6.2(5.2-8.3)$ \\
\hline Ottawa & $4(1)$ & $6.1(6.1-6.1)$ & $6.3(5.7-8.2)$ \\
\hline London & $6(1)$ & $7.2(7.2-7.2)$ & $6.1(5.5-7.8)$ \\
\hline Calgary & $244(38)$ & $5.4(5.0-6.3)$ & $5.9(5.1-8.1)$ \\
\hline \multicolumn{4}{|l|}{ Disease's characteristics } \\
\hline Disease duration $(n=623)$ & $10.1(2.7-20.6)$ & $0.11(p=0.241)$ & $0.07(p=0.106)$ \\
\hline Recent diagnosis ( $<=15$ months) & & NC & NC \\
\hline \multicolumn{4}{|l|}{ Ancient diagnosis (>15 months) } \\
\hline ACR Criteria Score $(n=626)$ & $5(4-6)$ & $0.29(p<0.001)$ & $0.30(p<0.001)$ \\
\hline ACR LSI Score $(n=626)$ & $5.8(5.1-7.9)$ & NC & NC \\
\hline SLICC Criteria Score $(n=538)$ & $6(5-8)$ & $0.49(p<0.001)$ & $0.42(p<0.001)$ \\
\hline SLEDAI score $(\mathrm{n}=598)$ & $2(0-6)$ & $0.05(p=0.586)$ & $-0.00(p=0.992)$ \\
\hline SLAQ Score $(n=407)$ & $12(7-18)$ & $-0.26(p=0.023)$ & $-0.18(p=0.001)$ \\
\hline SDI Score $(n=609)$ & $0(0-2)$ & $p=0.296$ & $p=0.005$ \\
\hline 0 & $310(51)$ & $5.5(4.8-6.4)$ & $5.6(4.9-7.9)$ \\
\hline$>0$ & $299(49)$ & $5.5(5.2-8.0)$ & $6.2(5.2-8.2)$ \\
\hline \multicolumn{4}{|l|}{ Medication } \\
\hline Prednisone $(n=614)$ & & $\mathrm{p}<0.001$ & $\mathrm{p}<0.001$ \\
\hline Yes & $203(33)$ & $6.4(5.3-8.2)$ & $6.8(5.5-8.2)$ \\
\hline No & $411(67)$ & $5.4(4.7-6.0)$ & $5.6(4.9-7.7)$ \\
\hline Prednisone dose $(n=219)$ & $7.5(5.0-15.0)$ & $0.41(p=0.001)$ & $-0.01(p=0.92)$ \\
\hline Imunomodulators ( $n=616$ ) & & $p=0.189$ & $p=0.028$ \\
\hline Yes & $554(90)$ & $5.5(5.0-6.9)$ & $5.9(5.2-8.1)$ \\
\hline No & $62(10)$ & $5.3(4.4-6.2)$ & $5.5(4.9-6.4)$ \\
\hline Antimalarial & & $p=0.241$ & $\mathrm{p}=0.97$ \\
\hline Yes & $517(83)$ & $5.5(4.9-6.8)$ & $5.8(5.1-8.1)$ \\
\hline No & $106(17)$ & $5.2(4.6-6.3)$ & $5.8(4.9-8.2)$ \\
\hline Biologic Agent $(n=609)$ & & $p=0.800$ & $p=0.270$ \\
\hline Yes & $25(4)$ & $5.9(5.3-6.5)$ & $6.1(5.3-8.4)$ \\
\hline No & $584(96)$ & $5.5(4.9-6.8)$ & $5.8(5.1-8.1)$ \\
\hline Death during follow-up & & NC & $p=0.002$ \\
\hline Yes & $12(2)$ & & $8.2(7.0-9.3)$ \\
\hline No & $627(98)$ & & $5.8(5.1-8.1)$ \\
\hline
\end{tabular}

${ }^{*} \mathrm{n}=639$ except when specified; ${ }^{*}$ Results presented as Median (1 st quartile 3rd quartile) or Frequency (percentage) for continuous and categorical variables respectively; ${ }^{* * *}$ Results presented as Spearman Correlation coefficient (pvalue) or Median (1 st quartile 3rd quartile) with Kruskal-Wallis pvalue for continuous and categorical variables respectively; NC: Not calculated. like disease activity and prednisone dose - modifiable. LSI can predict adverse outcome such as damage or death over time. Funding Source(s): Lupus Canada.

\section{MACROPHAGE ACTIVATION SYNDROME AS A PRESENTATION IN PEDIATRIC LUPUS: A RETROSPECTIVE STUDY OF 3 CASES}

${ }^{1}$ Ankita Singh* ${ }^{2}$ Rakesh Pilania, ${ }^{1}$ Sandesh Guleria, ${ }^{2}$ Nameirakpam Johnson, ${ }^{2}$ Gummadi Anjani, ${ }^{1}$ Ankur Jindal, ${ }^{2}$ Pandiarajan Vignesh, ${ }^{1}$ Deepti Suri, 'Surjit Singh. ${ }^{1}$ Postgraduate Institute of Medical Education and Research, Chandigarh, India; ${ }^{2}$ Dept. of Pediatrics, Allergy- Immunology Unit, Postgraduate Institute of Medical Education and Research

\subsection{6/lupus-2019-Ism.129}

Background Macrophage activation syndrome (MAS) can, at times, be the presentation of pediatric lupus and diagnosis requires high index of suspicion.

Methods We retrospectively studied 140 pediatric lupus patients from January 1993- November 2018 and collected clinical and laboratory data of patients (3) who had MAS as presenting manifestation.

Results Case 1 was 11-year-old girl with fever for 4 months associated with rash and generalized body swelling for 1 month. Examination revealed rash over malar area and ear lobules, anasarca, hepatomegaly, bilateral pleural and pericardial effusion. In view of multisystem involvement a possibility of lupus was considered which was confirmed by investigations (table 1). She had elevated ferritin and fasting triglyceride and low fibrinogen. A clinical possibility of lupus with associated MAS was considered. She received pulses of methylprednisolone, one dose of intravenous immunoglobulin following which she improved. In view of nephrotic range proteinuria she was started on induction regimen with cyclophosphamide and shifted to mycophenolate in maintenance. Her initial SLEDAI-2k was 32- this decreased to 4 at 3 year follow-up.

Case 2 was a 9-year-old girl with fever, rash, generalized body swelling for 1 month and altered sensorium for 4 days. Examination revealed pallor, oral ulcers and hepatomegaly. She was in shock at presentation. In view of multisystem involvement a possibility of lupus was considered which was confirmed by investigations (table 1). She had pericardial effusion and low ejection fraction (25\%). A possibility of MAS was considered and investigations revealed hyperferritinemia, elevated triglyceride and hypofibrinoginemia. She was given methylprednisolone pulses and continued on oral prednisolone, mycophenolate and hydroxychloroquine. Her initial SLEDAI$2 \mathrm{k}$ was 17 - this decreased to 0 at 3 year follow-up.

Case 3 was an 8-year-old girl who had fever, rash and body swelling for 15 days. She had tachycardia, tachypnea, pallor, anasarca, subconjunctival bleed and frontal alopecia. She had pleural and pericardial effusion. In view of multisystem involvement a possibility of lupus was considered which was confirmed by investigations (table1). She had high ferritin and triglyceride. So a possibility of MAS was considered and she received pulse methyl prednisolone and intravenous immunoglobulin. She also had hematuria and proteinuria (renal biopsy could not be performed as she was sick and had thrombocytopenia) so was given pulse cyclophosphamide 